UL-NTZ 08/97

HUB-EP-97/14

\title{
Measuring the interface tension when the electroweak phase transition becomes weak
}

\author{
M. Gürtler ${ }^{1 *}$ E.-M. Ilgenfritz ${ }^{2 \dagger}$ and A. Schiller ${ }^{1 \ddagger}$ \\ ${ }^{1}$ Institut für Theoretische Physik, Universität Leipzig, Germany \\ ${ }^{2}$ Institut für Physik, Humboldt-Universität zu Berlin, Germany
}

April 16, 1997

\begin{abstract}
We measure the interface tension near the phase transition endpoint of the $3 d S U(2)-$ Higgs model. The tunnel correlation length method is used and compared to other approaches. A modified scaling behaviour for the mass gap as function of the transverse area is proposed.
\end{abstract}

\section{Introduction}

It is now almost established that the symmetry restoring electroweak phase transition changes into a smooth crossover if the mass of the so far elusive Higgs particle would be equal to the $\mathrm{W}$-mass (or less within 10 per cent) [1, 2, 3, 画. This emerges from lattice studies in the effective 3-dimensional model as well as from 4-dimensional simulations, the latter so far only with a rough lattice spacing of order $O(1 /(2 T))$ with temperature $T$.

\footnotetext{
*guertler@tph204.physik.uni-leipzig.de

†ilgenfri@pha1.physik.hu-berlin.de

${ }^{\ddagger}$ schiller@tph204.physik.uni-leipzig.de
} 
In order to quantify the strength of the first order transition near to the critical Higgs mass several quantities have been considered. Measuring the discontinuity of the Higgs condensate is practically tantamount (in three dimensions) to calculating the latent heat. Both quantities are easy to obtain but are very sensitive to finite volume effects. It is even more difficult to measure the interface tension for weak transitions. This we have observed in our recent work [5] where we have estimated the interface tension for a Higgs mass near to its critical value.

In the literature mainly three methods are discussed to extract the interface tension from Monte Carlo studies. In the present work we shall employ and critically discuss the tunneling correlation length method [6, 7], which has been used so far only in the analysis of 4-dimensional simulations $[\mathbf{8}, 9]$ in the context of the electroweak phase transition.

In section 2 we define the model and discuss the methods used to extract the interface tension. The results obtained by the tunneling correlation method are presented and critically discussed in section 3. They are put into perspective with all available lattice measurements for arbitrary Higgs masses in section 4, which contains also our conclusion on the capability of the method.

\section{The Model and How to Measure the Interface Tension}

The model under study is a 3-dimensional $S U(2)$-Higgs system with one complex Higgs doublet of variable modulus. The gauge fields are represented by unitary $2 \times 2$ link matrices $U_{x, \alpha}$ and the Higgs fields are written as $\Phi_{x}=\rho_{x} V_{x}$. $\rho_{x}^{2}=\frac{1}{2} \operatorname{tr}\left(\Phi_{x}^{+} \Phi_{x}\right)$ is the Higgs modulus squared, $V_{x}$ an element of the group $S U(2), U_{p}$ denotes the $S U(2)$ plaquette matrix. The lattice action is

$$
\begin{aligned}
S= & \beta_{G} \sum_{p}\left(1-\frac{1}{2} \operatorname{tr} U_{p}\right)-\beta_{H} \sum_{l=(x, \alpha)} \frac{1}{2} \operatorname{tr}\left(\Phi_{x}^{+} U_{x, \alpha} \Phi_{x+\alpha}\right) \\
& +\sum_{x}\left(\rho_{x}^{2}+\beta_{R}\left(\rho_{x}^{2}-1\right)^{2}\right)
\end{aligned}
$$

(summed over plaquettes $p$, links $l$ and sites $x$ ), with the gauge coupling $\beta_{G}$, the lattice Higgs self-coupling $\beta_{R}$ and the hopping parameter $\beta_{H}$. To relate the lattice couplings to continuum quantities the notations of [5] are used.

Let us recall the procedures used for extracting the interface tension of the electroweak phase transition. The first one, the two-coupling method, requires 
a careful extrapolation to the limits $\beta_{H c} \pm \varepsilon$ of simulations in two coupled subvolumes [9]-12] supposed to be kept in the two phases. The variants differ in the way this limit is taken. In our version of the method [11] we have used a simultaneous multihistogram technique for a system consisting of two parts in order to estimate the free energy difference between the homogeneous and mixed states at $\beta_{H c}$.

The majority of results for the interface tension has been obtained with the second method based on the two-state signal in the histogram of an order parameter like quantity $o$ [10, 13, 5, 3]. Here the whole system is simulated at the pseudocritical point. The bulk variable under consideration is, say, the volume average of the modulus squared $o=\overline{\frac{1}{2} \operatorname{tr}\left(\Phi^{+} \Phi\right)}$ or the average link $o=\overline{\frac{1}{2} \operatorname{tr}\left(\Phi^{+} U \Phi\right)}$. Usually, the interface tension $\alpha$ is estimated comparing the minimum and the two maxima of the doubly peaked histogram $p(o)$ by

$$
\frac{\alpha a^{2}}{T_{c}}=\frac{1}{2 L^{2}} \log \frac{p_{\max }}{p_{\min }}
$$

where $L$ is a typical linear extension of a surface eventually dividing the system into different phases (the smaller extensions of a cylindrical system), $a$ denotes the lattice spacing and $T_{c}$ the corresponding transition temperature. Different entropy factors in the thermodynamic weight of the mixed state have been ignored for simplicity in (2), but are necessary to extract a reasonable estimate for the interface tension, in particular if histogram data of lattices with various geometries are used simultaneously [14]. Applying a formula like (2) one tacitly assumes that $(i)$ the order parameter $o$ is well-chosen in order to yield a histogram with clearly separated maxima and a broad minimum in between, (ii) the case of equal height of the maxima is near to the phase equilibrium, (iii) a minimal surface spanning through the lattice separates the pure phases from each other.

Lattice studies of the electroweak transition in the case of the Standard Model for realistic values of the Higgs mass are known to possess a transition which is very asymmetric and weak. Therefore the conditions $(i)$ to (iii) are hardly fulfilled. By "asymmetric" we mean that the fluctuations of suitable order parameters $o$ are stronger in one (the Higgs) phase than in the other (symmetric) phase. This fact makes it more and more difficult to use histogram methods based on (2) when approaching the critical Higgs mass.

In order to cope with overlapping histograms we have recently proposed a method to split histograms $p(o)$ into pure phase and mixed phase contributions [5] even under realistic circumstances of a very weak transition. This has enabled us to employ the histogram reweighting technique to find the pseudocritical coupling by the equal weight criterion. As a by-product, we have also obtained 
the latent heat from the variation of both pure phase thermodynamic weights near to the transition point and the thermodynamic weight of mixed states. Extracting, however, the interface tension from the relative weights of pure and mixed phases still depends on the simplifying assumption (iii) above. Collecting data from lattices with different aspect ratios and extrapolating in the smallest linear extension to infinity we [5] obtained at the physical Higgs mass $m_{H}=$ $64.77 \mathrm{GeV}$ (which corresponds to $T_{c}=150.9 \mathrm{GeV}$ ) for the $S U(2)$-Higgs theory without fermions an estimate of $\alpha / T_{c}^{3}=2.1 \times 10^{-4}$.

All methods discussed so far are focusing on changes of (volume or subvolume) averaged variables $o$ in mixed phase systems with minimal interfaces. There is a third method [6, 7] to deduce the interface tension $\alpha$ from a tunneling correlation length $\xi_{\text {tunnel }}$ at the phase transition. To be more precise, it is the dependence of this correlation length on the geometry of the system which allows to extract $\alpha$. A first test of the credibility of the method in the case of the electroweak phase transition has been undertaken in Ref. [8] far from the critical Higgs mass and in Ref. [9] at even smaller Higgs mass, both within the 4-dimensional framework.

In contrast to measurements of the temperature dependent Higgs mass on both sides of the transition [5] (which requires to separate pure phase samples) the emphasis is here on correlations due to different phases in coexistence. The tunneling correlation length is measured in a very elongated volume $L^{2} \times L_{z}$, stretched along the $z$-direction. In 4 -dimensional simulations the additional Euclidean temporal extent is understood to represent the (inverse) temperature and cannot be modified in practice. The connected correlator $C_{\text {conn }}\left(z_{1}-z_{2}\right)$ of $o(z)=\sum_{x} \delta_{x_{3}, z} \operatorname{tr}\left(\Phi_{x}^{+} \Phi_{x}\right)$ between two equal-z slices defines the correlation length $\xi_{\text {tunnel }}$ which is expected to vary with the transversal extent of the lattice as

$$
\xi_{\text {tunnel }} \propto \exp \left(\alpha_{3} A\right), \quad A=(a L)^{2}
$$

The interface tension of the original 4 -dimensional theory $\alpha$ is related to $\alpha_{3}$ through $\alpha=\alpha_{3} T_{c}$.

Using a semiclassical expansion for a scalar $\varphi^{4}$ theory in $3 d$ including quadratic fluctuations around a kink solution [7], the mass (energy) gap $m=\xi_{\text {tunnel }}^{-1}$ has been calculated (in lattice units) as

$$
m_{\text {lat }}=m a=C \sqrt{\frac{\alpha a^{2}}{T_{c}}} \exp \left(-\frac{\alpha L^{2} a^{2}}{T_{c}}\right)
$$

with no additional $L$ dependence in front of the exponent. Therefore, expressed 
in terms of the dimensionless parameter

$$
x=\frac{\alpha L^{2} a^{2}}{T_{c}}
$$

the following scaling behaviour is expected to hold for the tunneling correlation lengths (if they are measured in units given by the transverse lattice size)

$$
m_{\text {lat }} L=C \sqrt{x} \exp (-x)
$$

Strictly speaking, this result is valid for systems within the universality class of the Ising model, but it has been confronted with the 4-dimensional $S U(2)-$ Higgs model in Ref. [8]. In this first application in the electroweak context (at smaller Higgs mass and, consequently, higher $\alpha$ ) it has been shown that the simple perturbative one-loop result (11) is reached from above with increasing transverse lattice size and is valid only beyond $x \simeq 1$ (cf. Fig. 1 below). It has been argued already in 15 that higher order corrections may lead to a pre-exponential power in $L$ with an exponent different from zero in (仼) 7 . Concentrating on the roughening of the interface in a capillary wave model beyond the Gaussian approximation [17] it has been found that (41) gets a correction factor $\left(1+O\left(T_{c} /\left(\alpha L^{2} a^{2}\right)\right)\right)$.

Recall also that in all derivations it has been assumed that the tunneling correlation length is much larger than the typical correlation lengths in the pure phases. In our recent studies at a physical Higgs mass of roughly $65 \mathrm{GeV}$ 河 we have measured the Higgs correlation lengths near to the phase transition in the pure phases as $\xi_{\text {broken }} / a=13.40(41)$ and $\xi_{\text {symm }} / a=9.71(29)$ which are not so small compared to the tunneling correlation length $1 /\left(m_{\text {lat }} a\right)$ as one will see later. Furthermore we have visualised there a typical mixed-phase configuration which had very rough interfaces separating different phases.

Therefore, in order to extract an interface tension at this very weak first order transition (with very rough interfaces) we assume that the tunneling mass gap can be parametrised in a more general form as function of the transverse extent $L$

$$
m_{\text {lat }} L=c L^{\gamma} \exp \left(-\alpha_{\text {lat }} L^{2}\right),
$$

with the fit parameters $c, \gamma$ and $\alpha_{\text {lat }}$. Using the $3 d$ continuum gauge coupling $g_{3}^{2}=4 /\left(\beta_{G} a\right)$, we can put $\alpha_{\text {lat }}$ into relation to the $3 d$ dimensionless interface

\footnotetext{
${ }^{1}$ This has been confirmed in a recent two-loop calculation [16].
} 
tension $\alpha_{3} / g_{3}^{4}$ by

$$
\frac{\alpha_{3}}{g_{3}^{4}}=\left(\frac{\beta_{G}}{4}\right)^{2} \alpha_{\mathrm{lat}} .
$$

As a check of this assumption we have to compare the interface tension with the result of other methods when these are available.

\section{Results and Discussion}

The lattice model (2) is used as in [5], in particular with the same update algorithm as described there. We are dealing with the two cases corresponding to the Higgs masses $M_{H}^{*}=70$ and $M_{H}^{*}=57.4423 \mathrm{GeV}$ (denoted in the following by $57 \mathrm{GeV}$ ). These cases correspond to $\lambda_{3} / g_{3}^{2} \approx 0.095703$ and $\lambda_{3} / g_{3}^{2} \approx 0.0644457$, respectively, ( $\lambda_{3}$ is the $3 d$ continuum Higgs self coupling) via

$$
\frac{\lambda_{3}}{g_{3}^{2}}=\frac{1}{8}\left(\frac{M_{H}^{*}}{80 \mathrm{GeV}}\right)^{2} .
$$

The Higgs mass $M_{H}^{*}$ (in $\mathrm{GeV}$ ) differs numerically only slightly from the physical Higgs mass $m_{H}$ in the $4 d$ theory without top.

The smaller Higgs mass is chosen in accordance with the work [13] (in their notation referred to as $m_{H}^{*}=60 \mathrm{GeV}$ ). Correlation function measurements have beeN Taken after each 10th iteration. The maximum of the integrated autocorrelation time for this quantity was about 26 at the smaller Higgs mass and 16 at the larger Higgs mass (each in case of the largest measured transverse size).

To obtain the tunneling correlation length $\xi_{\text {tunnel }}$ requires some tuning. At first, the appropriate hopping parameter value $\beta_{H}$ has to be tuned, separately for each transverse size of the system while keeping near to the bulk critical value $\beta_{H c}$, to the maximum of the tunneling correlation length. The actual longitudinal size of the lattice has been chosen three to four times larger than the correlation length one is going to measure.

In the tables we quote the statistics for all lattice geometries $L^{2} \times L_{z}$ at the respective $\beta_{H}$ (having the maximal tunneling correlation length) and its corresponding value $m_{\text {lat }}$. We indicate also the inverse transverse correlation length $m_{\perp}$ lat and the total statistics which went into the search for the minimum. Additionally, we have checked at the larger Higgs mass for geometries $12^{2} \times 96$ and $20^{2} \times 128$ that $m_{\text {lat }}$ does not change within the errors for larger $L_{z}$. 


\begin{tabular}{|c|c|c|c|c|c|}
\hline$L^{2} \times L_{z}$ & $m_{\text {lat }}$ & $\#$ msmts & $\beta_{H}$ & $m_{\perp \text { lat }}$ & total \# msmts \\
\hline $4^{2} \times 32$ & $.2180(106)$ & 8000 & .343600 & - & 36000 \\
$6^{2} \times 64$ & $.1543(117)$ & 10000 & .343000 & - & 24000 \\
$8^{2} \times 64$ & $.1165(081)$ & 6000 & .342700 & - & 8000 \\
$10^{2} \times 64$ & $.09150(531)$ & 6000 & .342700 & $.1008(29)$ & 10000 \\
$12^{2} \times 64$ & $.07060(339)$ & 6000 & .342694 & $.0782(21)$ & 12000 \\
$14^{2} \times 96$ & $.05200(380)$ & 10000 & .342700 & $.0632(21)$ & 19000 \\
$16^{2} \times 128$ & $.04195(286)$ & 10000 & .342688 & $.0509(15)$ & 21000 \\
$18^{2} \times 128$ & $.03517(331)$ & 12000 & .342686 & $.0425(14)$ & 20000 \\
\hline
\end{tabular}

Table 1: Statistics at $M_{H}^{*}=57 \mathrm{GeV}$

\begin{tabular}{|c|c|c|c|c|c|}
\hline$L^{2} \times L_{z}$ & $m_{\text {lat }}$ & $\#$ msmts & $\beta_{H}$ & $m_{\perp \text { lat }}$ & total \# msmts \\
\hline $4^{2} \times 128$ & $.276(6)$ & 3000 & .345000 & - & 18400 \\
$6^{2} \times 64$ & $.1933(044$ & 10000 & .344200 & - & 60000 \\
$8^{2} \times 64$ & $.1489(053)$ & 20000 & .343800 & - & 40000 \\
$10^{2} \times 64$ & $.1174(043)$ & 25000 & .343540 & $.1345(17)$ & 80000 \\
$12^{2} \times 64$ & $.09530(278)$ & 50000 & .343540 & $.1080(13)$ & 110000 \\
$14^{2} \times 64$ & $.08031(267)$ & 25000 & .343540 & $.0889(16)$ & 25000 \\
$16^{2} \times 64$ & $.06910(154)$ & 25000 & .343540 & $.0761(09)$ & 40000 \\
$20^{2} \times 64$ & $.05367(163)$ & 15000 & .343560 & $.0599(12)$ & 51000 \\
\hline
\end{tabular}

Table 2: Statistics at $M_{H}^{*}=70 \mathrm{GeV}$

In order to extract the correlation length $\xi_{\text {tunnel }}$ we first check by inspection whether the local mass has a plateau. We define a local lattice mass $m_{\text {lat }}(z)$ at correlation distance $z$ through a fit of three subsequent values $C_{\text {conn }}(z-1)$, $C_{\text {conn }}(z)$ and $C_{\text {conn }}(z+1)$ of the correlation functions of $o(z)=\sum_{x} \delta_{x_{3}, z} \operatorname{tr}\left(\Phi_{x}^{+} \Phi_{x}\right)$ to a hyperbolic cosine shape $A\left(\exp \left(-m_{\text {lat }}(z) z\right)+\exp \left(-m_{\text {lat }}(z)\left(L_{z}-z\right)\right)\right)$. This ansatz is essential in order to observe a plateau in the local mass versus $z$. In Fig. $1 m_{\text {lat }}(z)$ is shown for one particular example. We contrast this with a local mass defined through a single exponential fit to the three neighbouring values of $C_{\text {conn }}(z)$ above. No plateau at all can be identified using the latter definition of a local mass. Finally, the (inverse of the) correlation length $\xi_{\text {tunnel }}$ is obtained by a global hyperbolic cosine fit over the plateau range that we have identified. The behaviour of the inverse correlation length near to its lowest value is demonstrated in Fig. 2.

In Fig. 3 we show the inverse tunneling correlation length multiplied by the transverse extension, $m_{\text {lat }} L$ as function of the transverse lattice area $L^{2}$ for both values of the Higgs mass under discussion. The strongly different exponential slopes reflect the weakening of the phase transition. The curves correspond to a least square fit with the ansatz (7). 


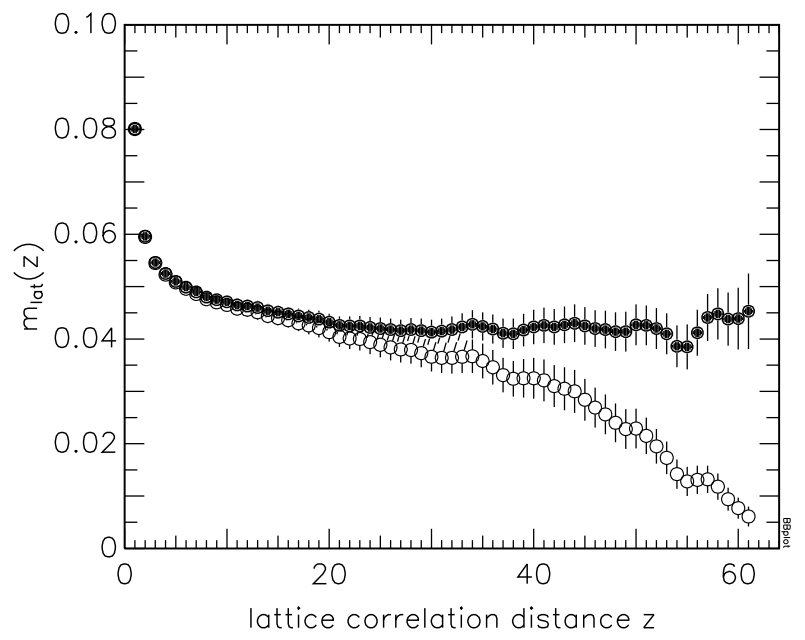

Figure 1: Example of the local lattice mass at $M_{H}^{*}=57 \mathrm{GeV}$ and $16^{2} \times 128$ as function of $z$

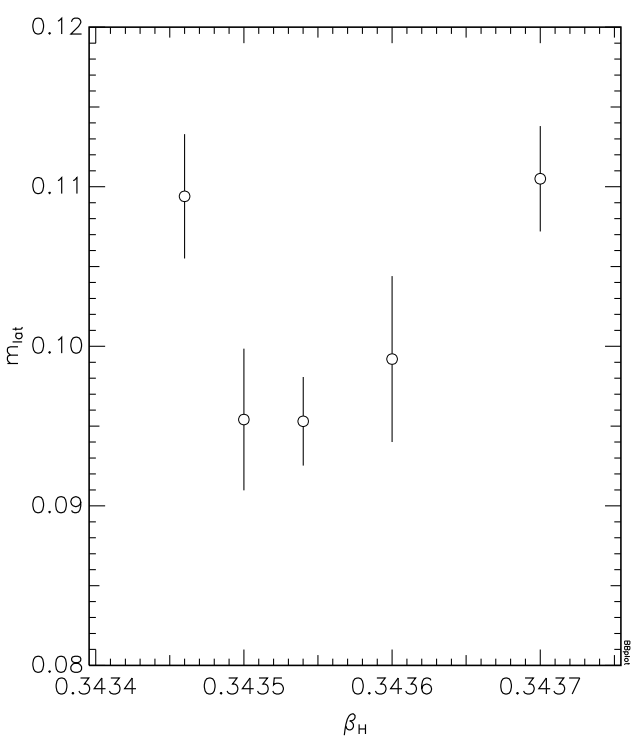

Figure 2: Inverse correlation length at $M_{H}^{*}=70 \mathrm{GeV}$ and $12^{2} \times 64$ as function of $\beta_{H}$

From this fit we extract the dimensionless $3 d$ interface tensions $\alpha_{3} / g_{3}^{4}=$ $0.0224(56)$ for the lower Higgs mass $M_{H}^{*}=57 \mathrm{GeV}$, and $\alpha_{3} / g_{3}^{4}=0.0049(18)$ 


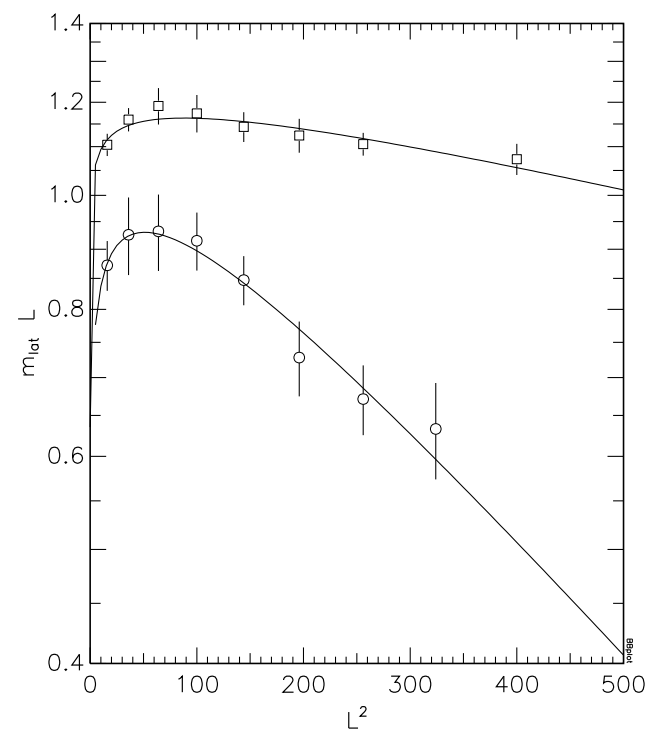

Figure 3: Fits for the inverse tunneling correlation lengths at $M_{H}^{*}=57 \mathrm{GeV}$ (circles) and $M_{H}^{*}=70 \mathrm{GeV}$ (squares)

for $M_{H}^{*}=70 \mathrm{GeV}$. The first number is in very good agreement with the value presented in Fig. 15 of [13] (and - with the use of eqs. (11.6) and (2.8) there translated into) $\alpha_{3} / g_{3}^{4}=0.0217(22)$.

Using these numbers we find the interface tensions $\alpha$

$$
\frac{\alpha}{T_{c}^{3}}=3.24(80) \times 10^{-3}, \quad M_{H}^{*}=57 \mathrm{GeV}
$$

and

$$
\frac{\alpha}{T_{c}^{3}}=70(26) \times 10^{-5}, \quad M_{H}^{*}=70 \mathrm{GeV}
$$

corresponding to the 4-dimensional model without top quark. Taking into account the top quark the numbers change to $3.57(89) \times 10^{-3}$ and $77(29) \times 10^{-5}$. The relations between 3-dimensional and 4-dimensional quantities have been obtained as in [5] using (in the same notations as there) table 3 which is recalculated here for $M_{H}^{*}=57 \mathrm{GeV}$. Though the top contribution apparently changes the interface tension only insignificantly $\left(\Delta_{g}\right.$ is small) some of the fermionic one-loop corrections to the $4 d$ couplings are already too large and the other 


\begin{tabular}{|r|r|r|}
\hline & $M_{H}^{*}=57 \mathrm{GeV}$ & $M_{H}^{*}=57 \mathrm{GeV}$ \\
& without fermions & with top \\
\hline$\Delta_{g}$ & -0.01322 & $6.712 \times 10^{-5}$ \\
$\Delta_{\lambda}$ & -0.02383 & 1.494 \\
$\Delta_{\nu}$ & -0.02938 & 0.9443 \\
$\Delta_{Y}$ & & -0.01301 \\
\hline
\end{tabular}

Table 3: Corrections in eq. (6) of [5]

physical numbers should be taken with great care. The reported pseudocritical $\beta_{H}$ values for the largest transverse sizes from tables 1 and 2 are related to critical temperatures $T_{c}=127.2$ and $150.1 \mathrm{GeV}$ and physical Higgs masses $m_{H}=52.35$ and $64.55 \mathrm{GeV}$ of the $4 d$ theory without top for the lower and larger $M_{H}^{*}$. The four dimensional $\overline{\mathrm{MS}}$ gauge coupling $g^{2}\left(m_{W}\right)$ has the value 0.423 and 0.422 , respectively, which is close to that of the standard model.

Our present result for the case of $M_{H}^{*}=70 \mathrm{GeV}$ is larger by a factor 3.3 than the previous rough estimate [5]. The latter was obtained as a result of our equal weight histogram method, finally based on a global infinite volume extrapolation of the mixed phase thermodynamical weight for lattices of various aspect ratios.

In the present fits we obtain effective exponents $\gamma=0.25(11)$ and $0.095(45)$ of $L$, respectively, which become smaller with decreasing strength of the transition. Fixing the exponent to $\gamma=1$ as suggested by eq. (4) we would be able to present only a local fit to the few highest transverse areas $L^{2}$. The interface tensions would be estimated by this fit as follows: $\alpha_{3} / g_{3}^{4}=0.023$ for $M_{H}^{*}=57 \mathrm{GeV}$ and 0.016 for $M_{H}^{*}=70 \mathrm{GeV}$. In the first case this would be still acceptable comparing with the result of the Helsinki group [13] for that Higgs mass. But the interface tension evaluated at $M_{H}^{*}=70 \mathrm{GeV}$ in this way does not follow the general trend of the interface tensions from $d=3$ simulations which have been collected in Ref. [18].

In view of the arguments above, we consider the fit with the free ansatz (7) more serious than the results of the fit confined to $\gamma=1$. We recall that also in Ref. [8] the latter fit has been successful only at large values of the scaling variable $x=\alpha L^{2} a^{2} / T_{c}$. The one-loop scaling law (6) was meant to hold independent of the particular system, irrespective of the actual value of the interface tension. With the values of $\alpha$ obtained now and in Refs. [8, 9] the underlying mass gaps for various Higgs masses and transverse lattice sizes seem to follow another universal law if expressed through the scaling variable $x$

$$
m_{\text {lat }} L=C^{\prime} \exp (-x)
$$




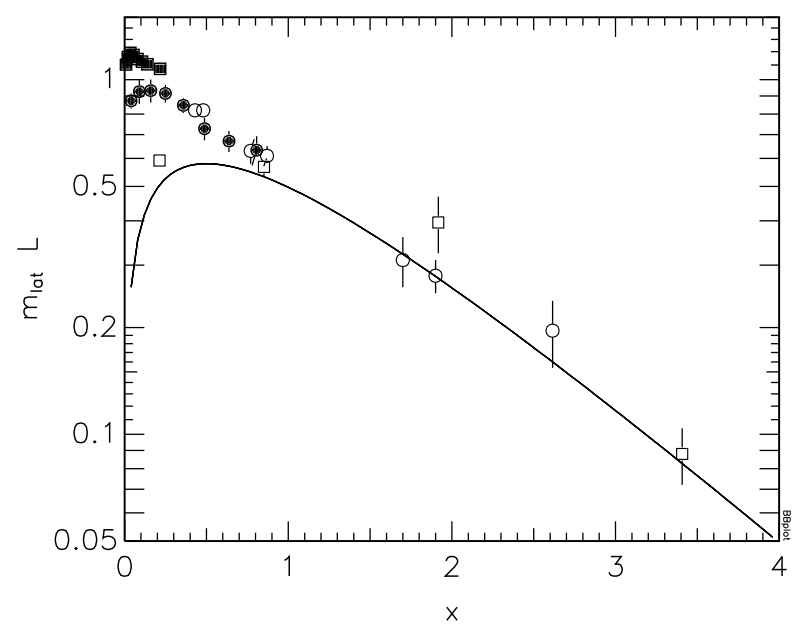

Figure 4: Scaling law for the mass gap followed by data at $m_{H}=35 \mathrm{GeV}$ (open squares) [9], $m_{H}=49 \mathrm{GeV}$ (open circles) [8], $M_{H}^{*}=57 \mathrm{GeV}$ (full circles) and $M_{H}^{*}=70 \mathrm{GeV}$ (full squares) [this work]

instead of (12), except for the smallest transverse extensions $L$ in each case. This can be seen in Fig. 4 . In this figure we also show the asymptotic behaviour (6) with $C=1.352$ as predicted by [7, 19$]$.

There is one warning in the data concerning the applicability of the analytical formulae used throughout the literature in order to extract the interface tension from lattice data of the tunneling correlation length. As mentioned above it is implicitly assumed that the correlation lengths of the two phases in equilibrium are small compared to the tunneling correlation length. From this point of view the present data on $m_{\text {lat }}$ for $M_{H}^{*}=70 \mathrm{GeV}$ (where we have measured Higgs masses separately for both phases at the transition temperature) indicate that transverse sizes $L \leq 10$ would have to be excluded from the analysis.

The other concern is caused by the fact that the transverse correlation length $\xi_{\perp}$ does not decouple from the transverse lattice extension at small $L$ and keeps growing for all transverse sizes considered. For instance, at $M_{H}^{*}=70 \mathrm{GeV}$, the transverse correlation length amounts from 75 to 83 per cent of $L$ on our lattices with transverse sizes $L \geq 10$ which are, on the other hand, the only acceptable ones in view of the criticism discussed before. In the case of $M_{H}^{*}=57 \mathrm{GeV}$ the transverse correlation length is even larger compared with the transverse size $L$ (100 to 130 per cent) on lattices with $L \geq 10$. It should be mentioned that the ratio of the transverse correlation length to the transverse size is consistent with the ratio of the bulk correlation length to the system size for cubic symmetries 
on top of the phase transition (measured without separating the Monte Carlo sample into pure phase configurations).

\section{Overview and Conclusions}

To compare our $3 d$ results for the interface tension with those of $4 d$ measurements we follow the procedure outlined in [20]. The measurements in the $4 d$ approach have been performed at a different gauge coupling. The measured renormalised gauge couplings do not seem to change significantly with the Higgs mass in the so far reported region from 18 to $49 \mathrm{GeV}$ [10, 21] and vary from 0.56 to 0.59 . For simplicity (and due to missing calculation) it is assumed as in [20] that the measured renormalised gauge coupling roughly corresponds to the $\overline{\mathrm{MS}}$ running coupling. It is then different from the value $g^{2}\left(m_{W}\right)=0.42$ corresponding to our calculations described in the last section.

Using the prescription to relate $3 d$ and $4 d$ parameters [5] we calculate Higgs masses, critical temperatures and interface tensions for increasing $4 d$ gauge couplings keeping the respective $\lambda_{3} / g_{3}^{2}$ fixed. The numbers are collected in table 4 . Note that the Higgs mass is slightly moving to lower values while the ratio $\alpha / T_{c}^{3}$

\begin{tabular}{|c|c|c|c|c|}
\hline$M_{H}^{*} / \mathrm{GeV}$ & $g^{2}\left(m_{W}\right)$ & $m_{H} / \mathrm{GeV}$ & $T_{c} / \mathrm{GeV}$ & $\alpha / T_{c}^{3}$ \\
\hline 57 & 0.423 & 52.35 & 127.2 & $0.00324(081)$ \\
& 0.560 & 50.86 & 108.2 & $0.00539(134)$ \\
& 0.570 & 50.75 & 107.1 & $0.00556(138)$ \\
& 0.580 & 50.64 & 106.0 & $0.00574(143)$ \\
& 0.590 & 50.54 & 104.9 & $0.00592(147)$ \\
\hline 70 & 0.422 & 64.55 & 150.1 & $0.00070(26)$ \\
& 0.560 & 62.99 & 128.1 & $0.00115(43)$ \\
& 0.570 & 62.88 & 126.8 & $0.00119(45)$ \\
& 0.580 & 62.76 & 125.5 & $0.00122(46)$ \\
& 0.590 & 62.65 & 124.3 & $0.00126(47)$ \\
\hline
\end{tabular}

Table 4: Higgs masses, critical temperatures and interface tensions for various $4 d$ running gauge couplings

becomes much bigger, largely due to the critical temperature getting smaller.

Having the caveats of the last section in mind we compare now in Fig. . interface tensions $\alpha$ with those measured by different methods in the $4 d$ theory at various Higgs masses as function of the physical Higgs mass $m_{H}$. In order to do 


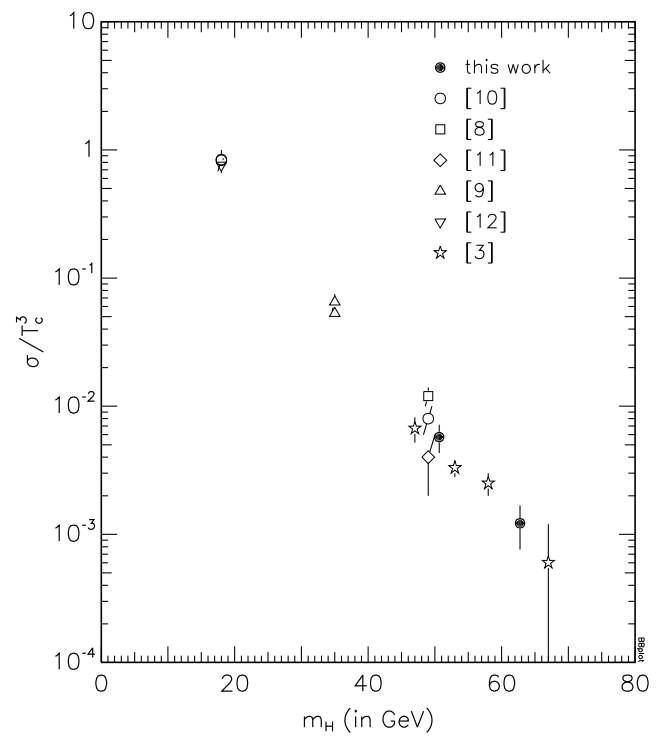

Figure 5: Collected results on the interface tension as function of the Higgs mass

this we read for our $3 d$ data (full points) the corresponding Higgs mass (without fermion contributions) from table 4 , for example at $g^{2}\left(m_{W}\right)=0.58$. This value has been deduced as mentioned above from the measured renormalised gauge coupling.

In the $4 d$ data the Higgs masses are either measured 10, 8, 11, 9, 12 (no errors are taken into account in the horizontal scale) or estimated [3]. The agreement between the various approaches in $3 d$ and $4 d$ is remarkable if the $3 d$ and $4 d$ parameters are correctly mapped onto each other. This nicely demonstrates the validity of dimensional reduction in the considered Higgs mass range.

Our new data points are the following ones, expressed in terms of the 3dimensional and the 4-dimensional interface tensions. We find at $\lambda_{3} / g_{3}^{2}=$ $0.0644457, g^{2}\left(m_{W}\right)=0.423$ and $\beta_{G}=12\left(M_{H}^{*}=57 \mathrm{GeV}\right)$

$$
\frac{\alpha_{3}}{g_{3}^{4}}=0.0224(56), \quad \frac{\alpha}{T_{c}^{3}}=3.24(80) \times 10^{-3}
$$

and at $\lambda_{3} / g_{3}^{2}=0.095703, g^{2}\left(m_{W}\right)=0.422$ and $\beta_{G}=12\left(M_{H}^{*}=70 \mathrm{GeV}\right)$

$$
\frac{\alpha_{3}}{g_{3}^{4}}=0.0049(18), \quad \frac{\alpha}{T_{c}^{3}}=70(26) \times 10^{-5}
$$


We observe an approximate scaling law expressing the energy gap for all Higgs masses in terms of the dimensionless variable $x$ (see eq. (5)) without the

prefactor $\sqrt{x}$ which had been suggested by one-loop perturbation theory for interfaces in the case of binary systems.

We emphasise that the tunneling correlation length method works well even near to the critical Higgs mass where other methods relying on discrimination of histogram peaks and minima are already difficult to apply.

\section{Acknowledgements}

E.M. I. is supported by the DFG under grant Mu932/3-4. We thank the council of HLRZ Jülich for providing CRAY-T90 resources.

\section{References}

[1] K. Kajantie, M. Laine, K. Rummukainen and M. Shaposhnikov, Phys. Rev. Lett. 77 (1996) 2887

[2] F. Karsch, T. Neuhaus, A. Patkós and J. Rank, Nucl. Phys. B(Proc. Suppl.) 53 (1997) 623

[3] Y. Aoki, Nucl. Phys. B(Proc. Suppl.) 53 (1997) 609 and hep-lat/9612023

[4] M. Gürtler, E.-M. Ilgenfritz and A. Schiller, in preparation

[5] M. Gürtler, E.-M. Ilgenfritz, J. Kripfganz, H. Perlt and A. Schiller, Nucl. Phys. B483 (1997) 383

[6] V. Privman and M.E. Fisher, J. Stat. Phys. 33 (1983) 385

[7] G. Münster, Nucl. Phys. B340 (1990) 559

[8] B. Bunk, Nucl. Phys. B(Proc. Suppl.) 42 (1995) 566

[9] F. Csikor, Z. Fodor, J. Hein, J. Heitger, Phys. Lett. B357 (1995) 156

[10] F. Csikor, Z. Fodor, J. Hein, K. Jansen, A. Jaster and I. Montvay, Phys. Lett B334 (1994) 405; Z. Fodor, J. Hein, K. Jansen, A. Jaster and I. Montvay, Nucl. Phys. B439 (1995) 147

[11] E.-M. Ilgenfritz and A. Schiller, Nucl. Phys. B(Proc. Suppl.) 42 (1995) 578

[12] J. Hein, J. Heitger, Phys. Lett. B385 (1996) 242 
[13] K. Kajantie, M. Laine, K. Rummukainen and M. Shaposhnikov, Nucl. Phys. B466 (1996) 189

[14] Y. Iwasaki, K. Kanaya, L. Kärkkäinen, K. Rummukainen and T. Yoshié, Phys. Rev. D49 (1994) 3540

[15] E. Brèzin and J. Zinn-Justin, Nucl. Phys. B257 (1985) 867

[16] G. Münster, private communication

[17] M. Caselle, R. Fiore, F. Gliozzi, M. Hasenbusch, K. Pinn and S. Vinti, Nucl. Phys. B432 (1994) 590

[18] K. Rummukainen, Nucl. Phys. B(Proc. Suppl.) 53 (1997) 30

[19] B. Bunk, Int. J. Mod. Phys. C3 (1992) 889

[20] M. Laine, Phys. Lett. B385 (1996) 249

[21] F. Csikor, Z. Fodor, J. Hein, A. Jaster and I. Montvay, Nucl. Phys. B474 (1996) 421 